\title{
Regulation of Zipcode Binding Protein 1 Transport Dynamics in Axons by Myosin Va
}

\author{
Vijayalaxmi C. Nalavadi, ${ }^{1}$ Laura E. Griffin, ${ }^{1}$ Philip Picard-Fraser, ${ }^{1}$ Andrew M. Swanson, ${ }^{1}$ Toru Takumi, ${ }^{3}$ \\ and Gary J. Bassell ${ }^{1,2}$ \\ Departments of ${ }^{1}$ Cell Biology and ${ }^{2}$ Neurology, Emory University School of Medicine, Atlanta, Georgia 30322, and ${ }^{3}$ Graduate School of Biomedical Sciences, \\ Hiroshima University, Hiroshima 734-8553, Japan
}

Directed transport of the mRNA binding protein, zipcode binding protein1 (ZBP1), into developing axons is believed to play an important role in mRNA localization and local protein synthesis. The role of molecular motors in this process is unclear. We elucidated a role for myosin $\mathrm{Va}(\mathrm{MyoVa})$ to modulate the axonal localization and transport of ZBP1 in axons. Using cultured rat hippocampal neurons, ZBP1 colocalized with MyoVa in axons and growth cones. Interaction of MyoVa with ZBP1 was evident by coimmunoprecipitation of endogenous and overexpressed proteins. Inhibition of MyoVa function with the globular tail domain (GTD) of MyoVa protein or short hairpin RNA led to an accumulation of ZBP1 in axons. Live cell imaging of mCherryZBP1 in neurons expressing GTD showed an increase in the number of motile particles, run length, and stimulated anterograde moving ZBP1 particles, suggesting that MyoVa controls availability of ZBP1 for microtubule-dependent transport. These findings suggest a novel regulatory role for MyoVa in the transport of ZBP1 within axons.

\section{Introduction}

Localized translation of mRNA transcripts serves to spatially and temporally control cell motility, developmental polarity during oogenesis and embryogenesis, axon guidance, and synaptic plasticity (Martin and Ephrussi, 2009). Transport of mRNA to distal compartments in neurons, such as growth cones and dendritic spines, ensures local translation of proteins in response to receptor signaling. mRNA binding proteins may serve as adapters to facilitate the microtubule-dependent transport of mRNA in dendrites and the actin-dependent anchoring of mRNA within dendritic spines (Yoshimura et al., 2006; Dictenberg et al., 2008). RNA binding proteins not only transport but also regulate translation of mRNAs (Kiebler and Bassell, 2006). Zipcode binding protein 1 (ZBP1) binds to a 54-nucleotide zipcode sequence in the $3^{\prime}$ untranslated region of $\beta$-actin mRNA, which is essential for mRNA localization in fibroblasts and neurons (Ross et al., 1997; Zhang et al., 2001). Neurotrophin signaling stimulates an increase in the transport of ZBP1 particles containing $\beta$-actin mRNA into developing axons and growth cones (Zhang et al., 2001). The Src kinase-dependent phosphorylation of ZBP1 removes translational inhibition (Hüttelmaier et al., 2005), which

\footnotetext{
Received April 25, 2012; revised Aug. 16, 2012; accepted Sept. 7, 2012.

Author contributions: V.C.N. and G.J.B. designed research; V.C.N., L.E.G., and P.P.-F. performed research; T.T. contributed unpublished reagents/analytic tools; V.C.N., L.E.G., P.P.-F., A.M.S., and G.J.B. analyzed data; V.C.N. and G.J.B. wrote the paper

This research was supported by NIH Grant MH085617 (to G.J.B.) and the Neuronal Imaging and Viral Vector Cores of the Emory Neuroscience NINDS Core Facilities (P30NS055077). We thank Yukio Sasaki for providing mCherry-ZBP1 constructs and anti-ZBP1 antibody and the Custom Cloning Core Facility, Emory University, for technical support.

Correspondence should be addressed to Gary Bassell, Departments of Cell Biology and Neurology, Whitehead Biomedical Research Building, Room 415, Emory University School of Medicine, 615 Michael Street, Atlanta, GA 30322. E-mail: gary.bassell@emory.edu.

DOI:10.1523/JNEUROSCI.2006-12.2012

Copyright $\odot 2012$ the authors $\quad 0270-6474 / 12 / 3215133-09 \$ 15.00 / 0$
}

is stimulated by BDNF in neurons to promote local translation of $\beta$-actin mRNA required for growth cone guidance (Yao et al., 2006; Sasaki et al., 2010; Welshhans and Bassell, 2011). Hence, the precise spatiotemporal control of ZBP1 localization is crucial for neuronal functions.

The "tug-of-war" model for bidirectional cargo transport proposes a coordination between opposing motors moving on microtubules (Ross et al., 2008; Hendricks et al., 2010; Holzbaur and Goldman, 2010) as well as actin (Ali et al., 2011) networks. In vitro and in vivo measurement of defined cargoes and motors indicated that opposing motors are simultaneously engaged on cargoes that undergo bidirectional transport and suggest a potential for regulation during activation by controlling motor type and number. It could be proposed therefore that directed ZBP1 transport is carried out by and requires a fine-tuned regulation of multiple microtubule and actin motors. Myosin $\mathrm{Va}(\mathrm{MyoVa})$ is a fast processive myosin that transports diverse cargoes such as vesicles (Wu et al., 1997; Kögel et al., 2010), neurofilaments (Rao et al., 2002), mitochondria (Pathak et al., 2010), and ER (Wagner et al., 2011), as well as RNA (Yoshimura et al., 2006; Salerno et al., 2008; Krauss et al., 2009). Mutations of MyoVa cause Griscelli syndrome in humans and dilute-lethal phenotype in mice that display defects in skin/coat color and have neurological phenotypes (Pastural et al., 1997; Takagishi and Murata, 2006). While MyoVa is important for neuronal development (Lewis et al., 2009; Tamada et al., 2010), its contribution to the trafficking of mRNA binding proteins important for mRNA localization is not well understood. We hypothesized that the prominent brain myosin MyoVa can modulate the axonal localization and transport of ZBP1. Our results indicate that MyoVa plays an inhibitory role and thus regulates $\mathrm{ZBP} 1$ accumulation in axons of cultured hippocampal neurons. MyoVa inhibition resulted in increased 


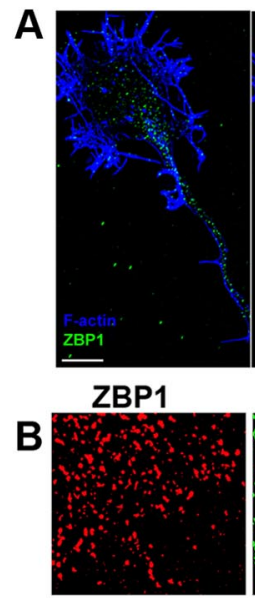

E

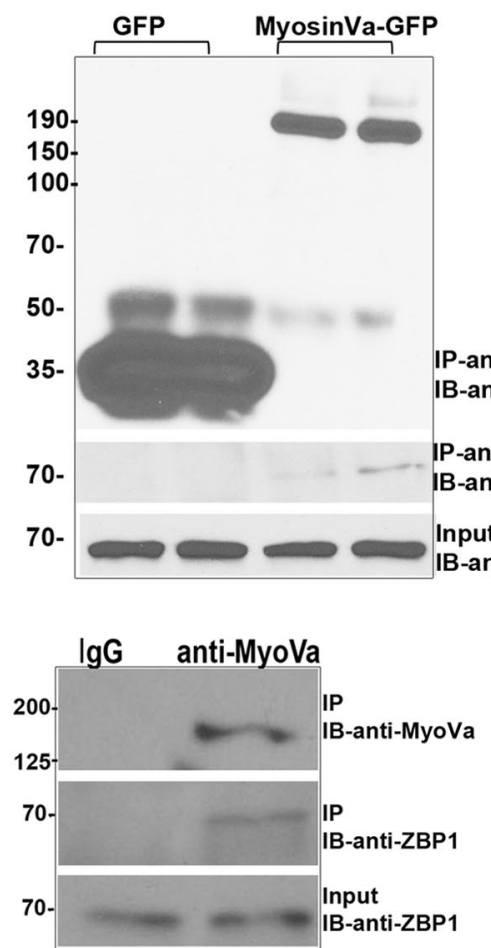

C

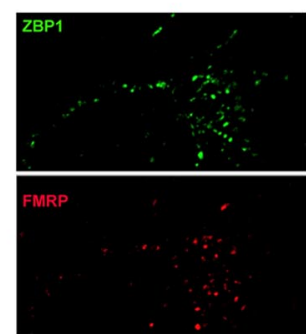

merged

MyoVa
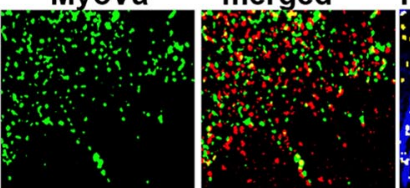

F-actin/coloc

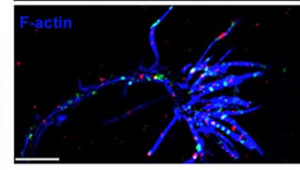

tै

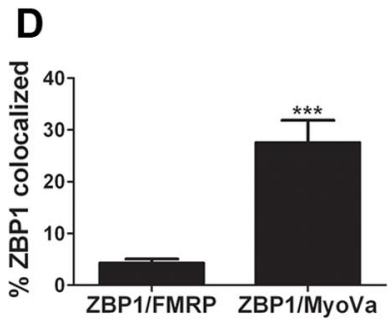

G mCherry mCherry mCherry ZBP1 ZBP1 -RNase +RNase

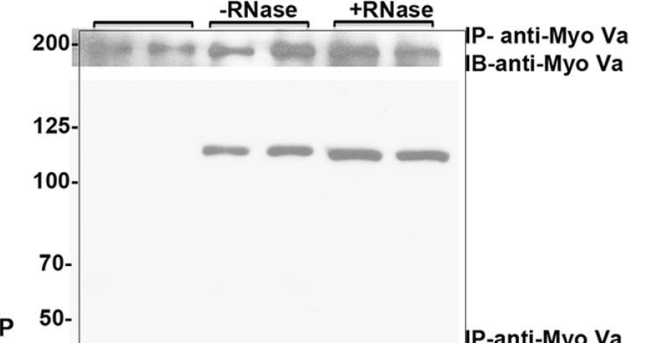

P-anti-Myo Va

IB- anti-ZBP1

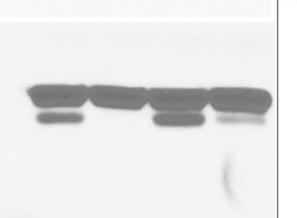

$70-$

50

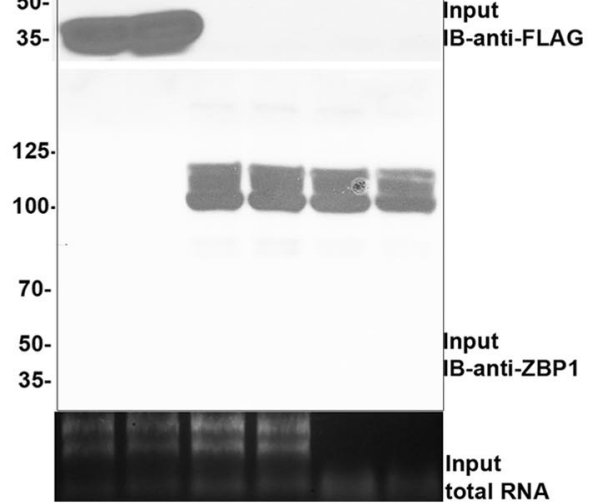

Figure 1. ZBP1 associates with MyoVa in neurons. A, Double-label immunofluorescence of ZBP1 (first panel on left) and MyoVa (second panel) and their colocalization in distal axon and growth cone of cultured hippocampal neuron (third and fourth panels). Neuronal morphology is shown by F-actin staining. The third panel shows an overlap of MyoVa and ZBP1 channels with the colocalized channel, and the fourth panel depicts the overlap of the colocalized channel with F-actin staining. B, Double-label immunofluorescence image of ZBP1 and MyoVa in a subregion of the growth cone shown in $A$, displayed at the automatic thresholds used for colocalization analysis by ImarisColoc (Bitplane). Neuronal morphology is shown by F-actin staining. C, Double-label immunofluorescence staining of ZBP1 and FMRP and their overlap with F-actin in a growth cone. $\boldsymbol{D}$, Histogram represents mean \pm SEM. of percent colocalization of ZBP1 with MyoVa or FMRP in deconvolved and 3D reconstructed Z-stacks from multiple growth cones $\left(n=3,{ }^{* * *} p<0.0001, t\right.$ test). $E$, Immunoblot (IB) screened with anti-ZBP1 antibody or anti-GFP antibody in duplicates of immunoprecipitates with an anti-GFP antibody of Neuro2a cell lysates expressing GFP alone or GFP-MyoVa. Position of molecular weight standards in kilodaltons is indicated on the left. $\boldsymbol{F}$, Immunoblots screened with anti-ZBP1 or anti-MyoVa antibody of immunoprecipitate with anti-MyoVa antibody or IgG from DIV3 high-density cortical neurons. Position of molecular weight standards in kilodaltons is indicated on the left. G, Immunoblots screened with anti-FLAG antibody in duplicates of immunoprecipitate with anti-MyoVa antibody of Neuro2a cells transfected with FLAG-mCherry alone or FLAG-mCherry-ZBP1 with or without treatment with RNase. Protein input was screened by immunoblotting with anti-FLAG and anti-ZBP1 antibodies. Lower panel shows an RNA gel of RNA extracted from the lysate post-RNase treatment. Position of molecular weight standards in kilodaltons is indicated on the left. 
A

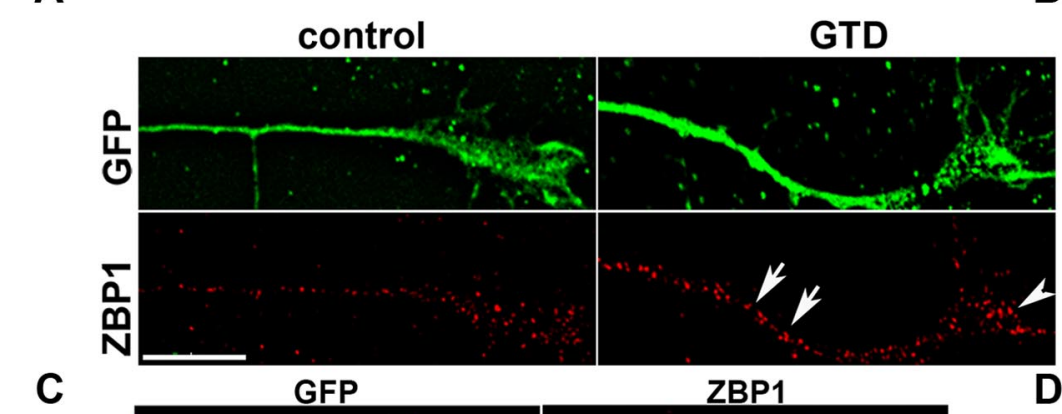

C

ZBP1

$\mathbf{E}$

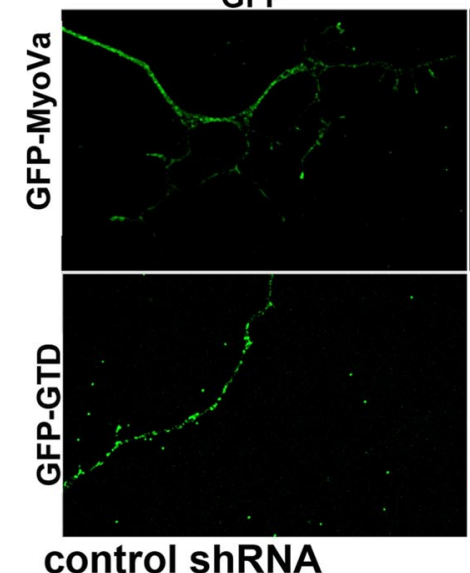

MyoVa shRNA
B

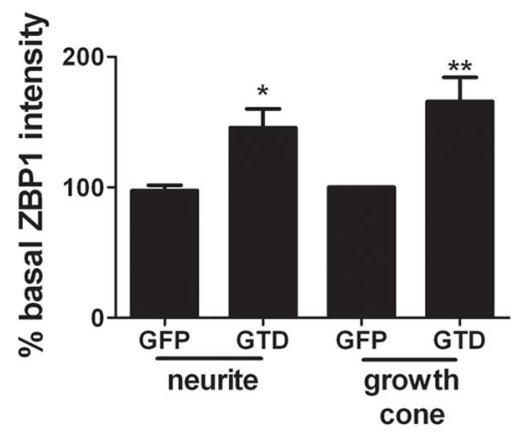

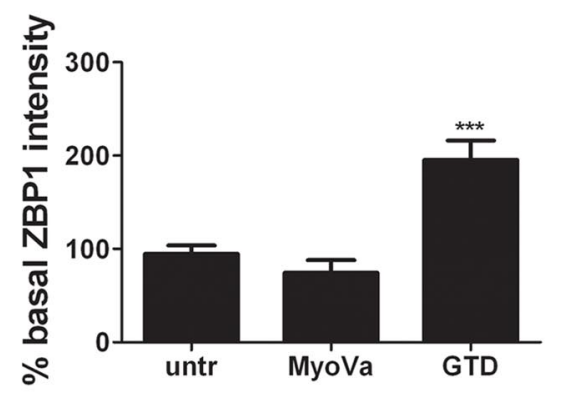

$\mathbf{F}$
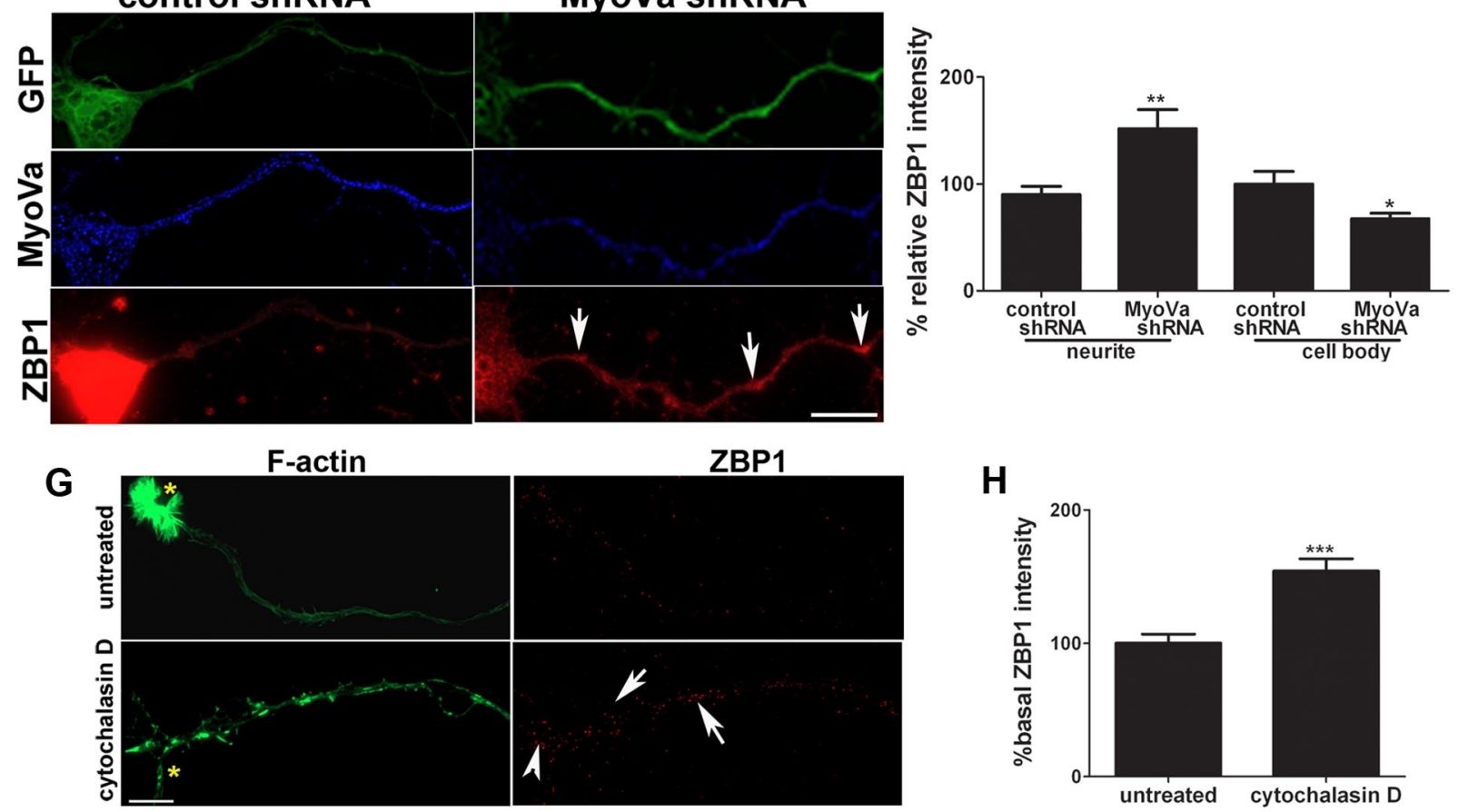

Figure 2. Inhibition of MyoVa function results in increased accumulation of ZBP1 in neurites. $A$, Images showing GFP and ZBP1 immunofluorescence staining of DIV3 rat hippocampal neurons transduced with GFP only or GFP-GTD for 12-16h. Arrows (axon) and arrowheads (growth cone) indicate increased ZBP1 staining in GTD-expressing neurons. $\boldsymbol{B}$, Histogram represents mean \pm SEM of normalized ZBP1 intensity in axons and growth cones of neurons expressing GFP alone or GFP-GTD $\left(n=3, p=0.0084\right.$, one-way ANOVA, Bonferroni, $\left.{ }^{*, * *} p<0.05\right)$. C, Images showing GFP and ZBP1 immunostaining of untransfected neurons or neurons transfected with GFP-GTD or GFP-MyoVa. Arrows indicate increased ZBP1 staining in GTD-expressing neurons. $\boldsymbol{D}$, Histogram shows mean \pm SEM. of ZBP1 intensities in multiple untransfected (untr) and transfected neurons $\left(n=3,{ }^{* * *} p<0.0001\right.$, one-way ANOVA, Bonferroni). E, Immunofluorescence images of ZBP1 and MyoVa staining in DIV3 neurons cotransfected with a construct expressing only GFP and a construct encoding control shRNA or MyoVa shRNA for $48 \mathrm{~h}$. GFP signal was used to identify transfected neurons. Arrows indicate increased ZBP1 staining in MyoVa-depleted neurons. $F$, Histogram representing mean \pm SEM of percent ZBP1 intensities relative to control shRNA in neurites and cell bodies of control shRNA- and MyoVa shRNA-transfected neurons ( $n=3, p=0.0003$, one-way ANOVA, Bonferroni, $\left.{ }^{*} * * * 0.05\right)$. G, Immunofluorescence images of ZBP1 staining in DIV3 neurons with or without treatment with cytochalasin-D. Arrows (axon) and arrowheads (growth cone) indicate increased ZBP1 staining in cytochalasin D-treated neurons. Yellow asterisk indicates the position of the growth cone. F-actin staining with Alexa Fluor 488-phalloidin was used to identify neuronal morphology as well as F-actin integrity. $\boldsymbol{H}$, Histogram represents mean \pm SEM of percent ZBP1 intensities in untreated or cytochalasin D-treated neurons $\left(n=3,{ }^{* * *} p<0.0001, t\right.$ test $)$. 

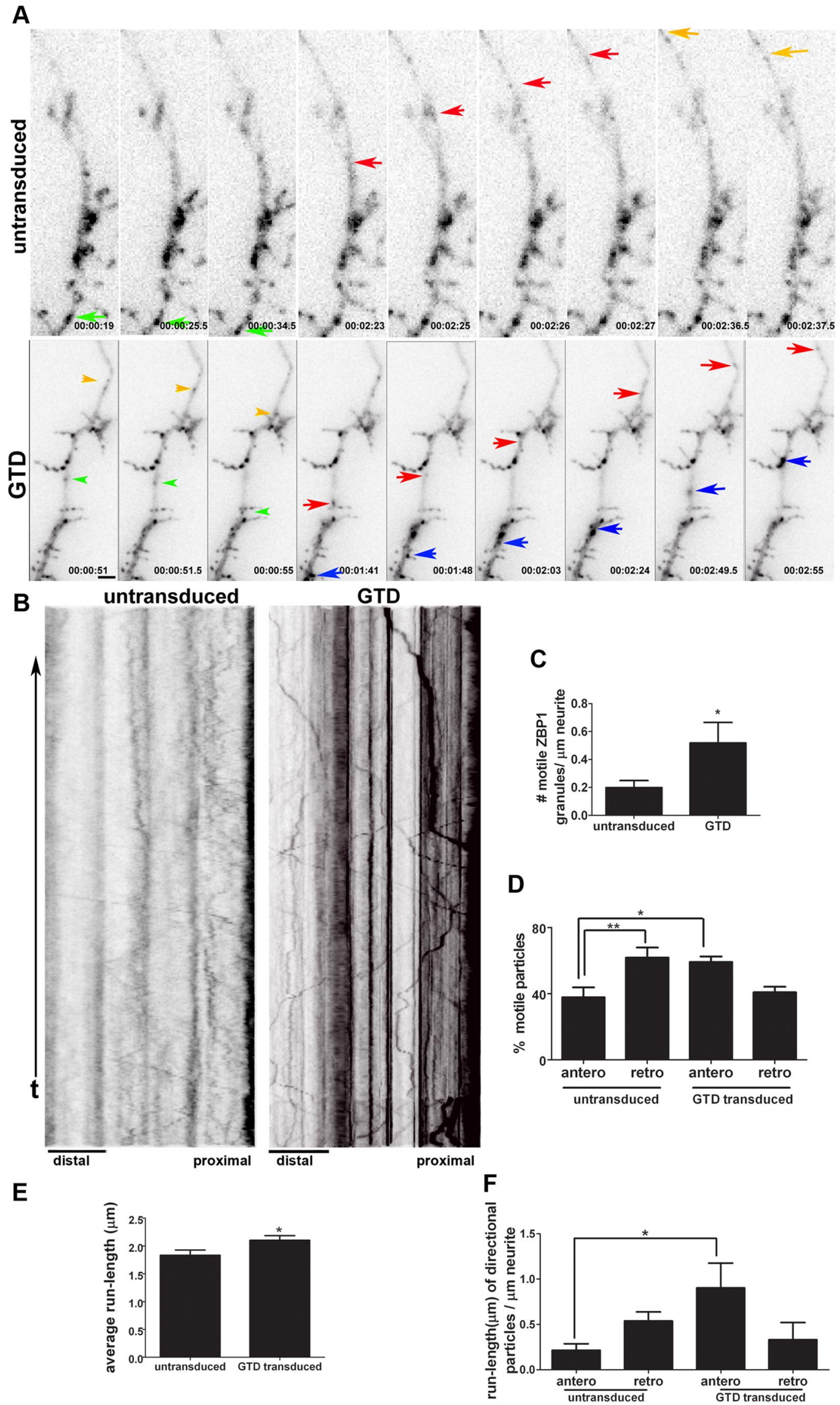

Figure 3. Inhibition of myosin function increases number of motile ZBP1 particles and changes orientation and run length. A, Sequential time lapse images of neurites of rat cortical neurons transfected with $\mathrm{mCherryZBP1}$ for 3 days without or with transduction with lentiviral vector expressing GFP-GTD. Arrows indicate retrograde (orange and green) or anterograde (Figure legend continues.) 
transport dynamics and reversal of orientation of ZBP1 particles in neurons favoring anterograde movement.

\section{Materials and Methods}

Cell culture. Hippocampal and cortical neurons were prepared from rat embryonic day 18 embryos of either sex as done previously (Sasaki et al., 2010).

DNA constructs and transfection. MyoVa globular tail domain (GTD) was amplified from mouse cDNA using the primers 5 '-CCAAGCTTTGCATCTTGAGGTCGCAGCTGGTGAGCCAAAAAGAAGCCATCC 3 - $^{\prime}$ and 5'-CGGAATTCTTATCAGTAGCTCACTGGAAACAACTGTTCATATTCTGAGG AGAAATGC-3' and cloned into HindIII-EcoRI sites of the plasmid pEGFPC1. An Age I-EcoRI fragment from this was cloned into corresponding sites in pFUGW to generate a lentiviral transfer vector and produce lentivirus expressing MyoVa GTD fused to green fluorescent protein (GFP). Lentiviruses were prepared at the Emory Neuroscience NINDS Core Facilities viral vector core facility. Photoactivatable (PA) green fluorescent protein-tagged GFP (PAGFP-ZBP1) was generated by replacing $3 \times$ FLAGmCherry with PAGFP in the $3 \times$ FLAGmCherryZBP1 vector (Sasaki et al., 2010). MyoVa-GFP, MyoVa short hairpin RNA (shRNA), and control shRNA containing vectors have been described previously (Yoshimura et al., 2006). Neurons were transfected by either nucleofection (Amaxa) or magnetofection (OZ Biosciences) according to the manufacturer's instructions. For nucleofection, $5 \times 10^{6}$ cells were used for electroporation with 3-5 $\mu \mathrm{g}$ of DNA containing mCherryZBP1(Sasaki et al., 2010) for particle tracking or PAGFP-ZBP1, either control shRNA or MyoVa shRNA, and mCherry for identification of transfected cells for live cell imaging with PAGFP-ZBP1. For magnetofection, day in vitro 1 (DIV1) rat hippocampal neurons were incubated on a magnetic plate (OZ Biosciences) for 20 min with DNA-magnetic bead (NeuroMag) complexes formed with either control shRNA or MyoVa shRNA and pMaxGFP to identify transfected neurons. The complexes were washed and neurons were incubated for $48 \mathrm{~h}$.

Immunofluorescence and image analysis. Transfected or transduced hippocampal neurons (DIV3) were fixed in $4 \%$ formaldehyde and labeled by immunofluorescence (IF) with guinea pig anti-ZBP1 antibody (Sasaki et al., 2010) and anti-MyoVa antibody. Multiple Z-slices of neurons were imaged on a Nikon Eclipse T500 inverted microscope equipped with a motorized stage with a $60 \times$ differential interference contrast (DIC) oil objective. The longest $(>50-100 \mu \mathrm{m})$ process having an axon-like morphology (DIV3) was analyzed for each neuron. Immunofluorescence (IF) signal intensity for ZBP1 was measured in a distal $(>50 \mu \mathrm{m})$ axonal segment using ImageJ. To disrupt F-actin, cytochala$\sin \mathrm{D}(5 \mu \mathrm{g} / \mathrm{ml})$ was added $30 \mathrm{~min}$ before fixation. For quantification of colocalization, analysis was carried out on growth cones of multiple 3D slices in the ImarisColoc module (Bitplane). Briefly, the algorithm calculated an automatic threshold for each of the channels by randomizing pixels from one channel and verifying that $>95 \%$ of the randomized iterations do not have a Pearson's correlation coefficient value greater than the original channel.

Live cell imaging and analysis. Rat cortical neurons were transfected with mCherryZBP1 at DIV0 by nucleofection and cultured on glassbottomed dishes (Bioptechs). After 2 days in culture, they were trans-

\section{$\leftarrow$}

(Figure legend continued.) (red and blue) orientation in an untransduced or GFP-GTDtransduced neurons. $\boldsymbol{B}$, Kymographs are shown from a 3 min movie with 360 frames from untransduced or GTD-transduced neurons. C, Histogram represents mean \pm SEM of number of motile ZBP1 particles per micrometer of neurite in multiple untransduced or GTD-transduced neurons $\left[n\right.$ (no. neurons $=15$, no. particles $=400-500$ ), ${ }^{*} p=0.025, t$ test $]$. D, Histogram represents mean \pm SEM percentage of ZBP1 particles in anterograde or retrograde orientation $\left(p=0.0029 ;{ }^{* *},{ }^{*} p<0.05\right.$, one-way ANOVA, Bonferroni) from multiple neurons untransduced or transduced with GTD. E, Histogram represents mean \pm SEM of average run lengths of total tracked ZBP1 particles (directed, bidirectional, and oscillatory) in untransduced neurons or neurons transduced with GFP-GTD ( ${ }^{*} p=0.045, t$ test). $\boldsymbol{F}$, Histogram represents mean \pm SEM of average anterograde or retrograde run-lengths of ZBP1 particles showing persistent, directional movement/micrometer of neurite in untransduced or neurons transduced with GFP-GTD ( ${ }^{*} p=0.0268$, one-way ANOVA, Bonferroni). duced with lentiviral vectors expressing GFP-GTD for 12-16 h. At DIV3, neurons expressing only mCherryZBP1 or mCherryZBP1 and GFP-GTD were imaged on a Nikon TE2000 inverted widefield microscope using a $60 \times$ DIC oil objective every $500 \mathrm{~ms}$ for $3 \mathrm{~min}$. ZBP1 particles were tracked using ImarisTrack (Bitplane), where mCherryZBP1 particles were used to build spots, and, after filtering background, the spots were automatically tracked over time applying an algorithm for autoregressive motion. Tracks with a defined straightness $(0.8)$ and gaps ( $<3$ frames) were selected and the statistics were extracted. The orientation of the spots was measured manually by counting directional particles moving toward the cell body as retrograde and away from the cell body as anterograde. In other experiments, PAGFP-ZBP1 with mCherry and either MyoVa shRNA or control shRNA were used to transfect cortical neurons by nucleofection. Transfected cells were identified by mCherry fluorescence, and the cell body was selectively photoactivated by a $405 \mathrm{~nm}$ laser at $40 \%$ power for 7 pulses of $2 \mathrm{~s}$ each on a Nikon A1R laser confocal microscope. Cells were imaged for 5 min every $2 \mathrm{~s}$, and the GFP fluorescence that accumulated in the nonactivated neurite was measured using NIS-Elements (Nikon). After background subtraction, data from multiple neurites were normalized as fold increase over basal GFP intensity of that neurite.

Immunoprecipitation and immunoblotting. Neuro2a cells transfected with either GFP or MyoVa-GFP and in other experiments with FLAGtagged mCherry or FLAG-tagged mCherry-ZBP1 for $24 \mathrm{~h}$ were used for immunoprecipitation using an anti-GFP antibody (Abcam) or an antiMyoVa (Sigma) antibody, respectively. DIV3 cultured high-density cortical neurons were used for immunoprecipitation with anti-MyoVa antibody (Sigma). The immunoprecipitates were screened by Western blot with an anti-ZBP1 antibody or an anti-FLAG antibody. For RNase treatment, cell lysates were treated with $2 \mu \mathrm{g} / \mathrm{ml}$ RNase A for $30 \mathrm{~min}$ at $37^{\circ} \mathrm{C}$ before incubation with anti-MyoVa antibody.

\section{Results}

ZBP1 associates with myosin Va in axons and growth cones of cultured neurons

To examine whether ZBP1 and MyoVa were colocalized in neurons, high-resolution immunofluorescence imaging experiments were performed to visualize endogenous MyoVa and ZBP1 in cultured hippocampal neurons (DIV3). Automated analysis of colocalization using ImarisColoc (Bitplane) revealed $27.5 \pm$ 9.2\% of ZBP1 particles colocalized with MyoVa in growth cones. Colocalized particles could be detected in neurites, filopodia, and lamellipodia of growth cones (Fig. $1 A, B, D$ ). As a negative control, colocalization between ZBP1 and Fragile X mental retardation protein, FMRP, another nonoverlapping (Narayanan et al., 2007) RNA binding protein that does not bind $\beta$-actin RNA (Muddashetty et al., 2007), was very low or not detected (4.2 \pm 0.76) (Fig. 1C,D). To further assess a possible interaction between MyoVa and ZBP1, we performed immunoprecipitation (IP) of MyoVa using multiple strategies. Endogenous ZBP1 was detected following immunoprecipitation of GFP-MyoVa using anti-GFP antibody in Neuro2a cells transfected with GFP-MyoVa and not in cells with GFP alone (Fig. 1E). Similarly, endogenous ZBP1 was also detected following immunoprecipitation of endogenous MyoVa using anti-MyoVa antibody applied to high-density cortical neurons (Fig. 1F). To investigate whether the interaction of ZBP1 and MyoVa might be dependent on an indirect interaction through RNA, we performed MyoVa IP experiments with lysates of Neuro2a cells expressing FLAG-mCherryZBP1 with or without pretreatment with RNase A. ZBP1 was detected in IPs of both RNase-treated and untreated cells, suggesting an interaction between MyoVa and ZBP1 that may be independent of RNA binding (Fig. 1G). 
A mCherry

PAGFP-ZBP1
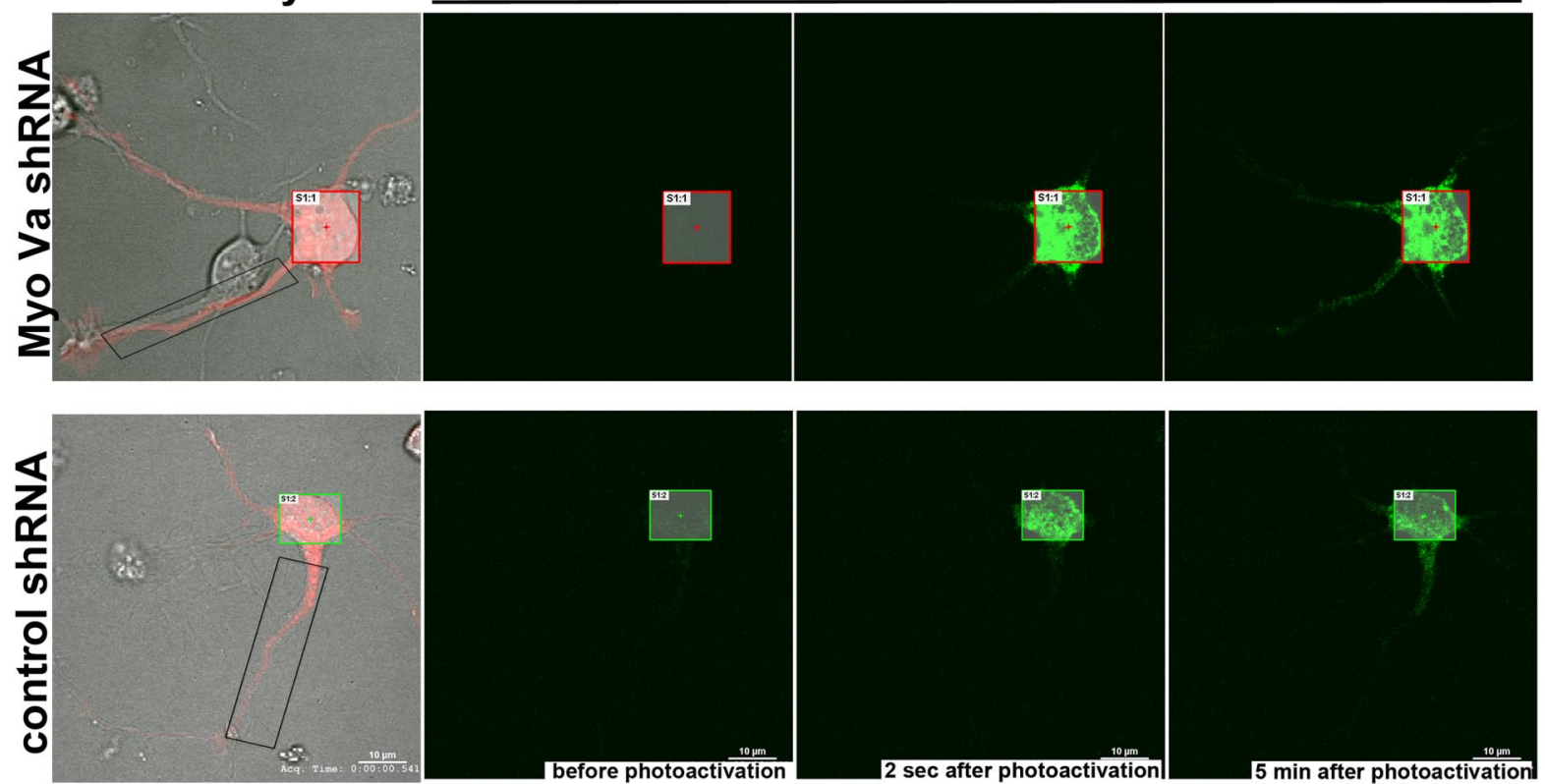

\section{B MyoVa shRNA}

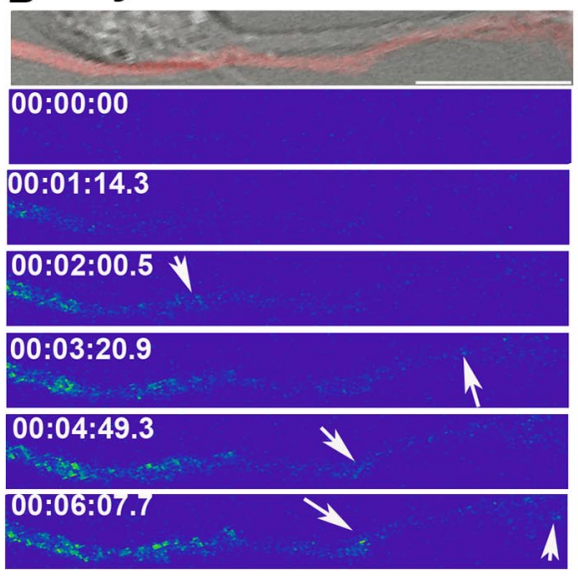

control shRNA

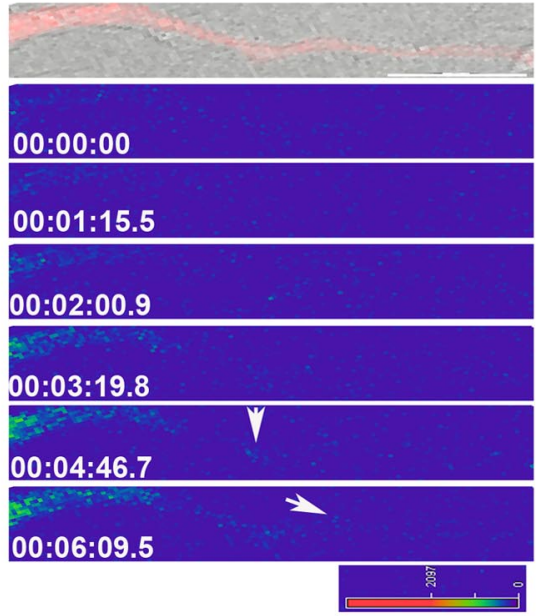

C

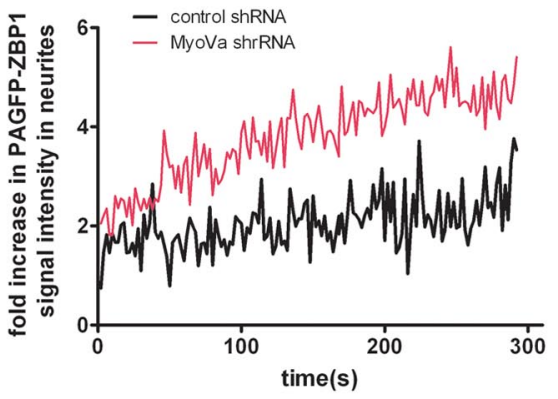

D

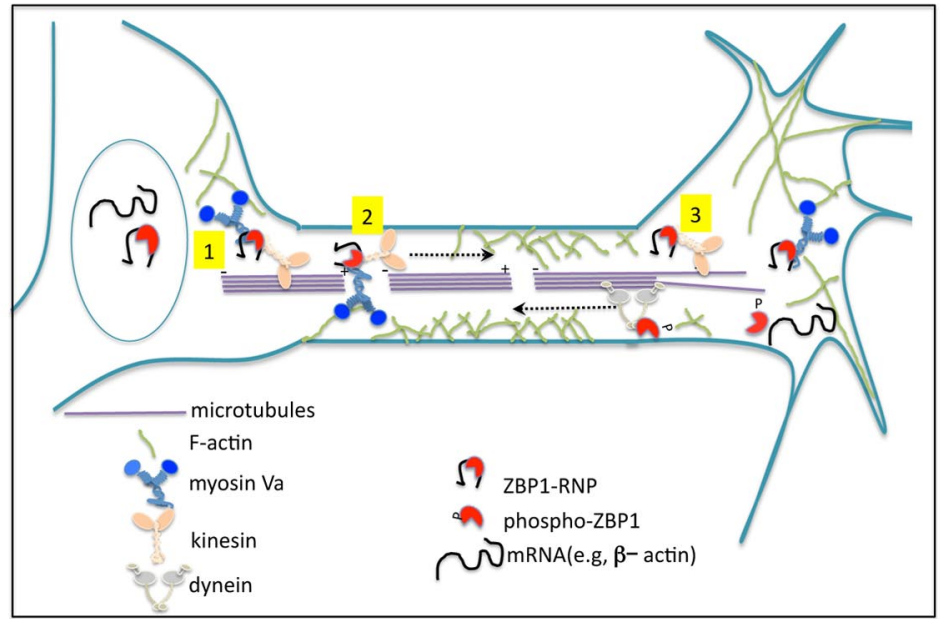

Figure 4. Inhibition of MyoVa leads to increased anterograde motility of ZBP1 from the cell body.A, Images showing cortical neurons transfected with mCherry and PAGFP-ZBP1 and with MyoVa shRNA or control shRNA at time 0 (first panel on left), before photoactivation (second panel), 2 safter photoactivation (third panel), and 5 min after photoactivation (fourth panel) of the cell body indicated by red and green boxes in MyoVa- or control shRNa-treated neurons, respectively. B, Sequential time-lapse images of a heat map of PAGFP-ZBP1 intensities accumulated in neurites marked by black boxes in neurons transfected with MyoVa shRNA and control shRNA in $\boldsymbol{A}$. Arrows indicate accumulated PAGFP-ZBP1 signal in distal neurites. $\boldsymbol{C}$, Histogram shows (Figure legend continues.) 
Myosin Va restricts $Z B P 1$ localization and regulates its dynamics in axons

The globular tail domain, GTD, of MyoVa has been used as an inhibitor of myosin function because it lacks the motor domain and acts as a dominant-negative construct (Li et al., 2006). Mouse brain cDNA was used to amplify the GTD and generate lentiviral vector particles expressing GTD. High-resolution imaging and quantitation of endogenous ZBP1 immunofluorescence intensities in axons and growth cones of hippocampal neurons transduced with GFP-GTD showed $45.7 \pm 14.4$ and $65.7 \pm 18.6 \%$ increases in ZBP1 signal, respectively, compared to GFP-only transduced neurons (Fig. $2 A, B$ ). Transfection of neurons with the full-length GFP-MyoVa did not change the levels of ZBP1 in axons, whereas transfection with GFP-GTD construct resulted in a $\sim 2$-fold increase in the axonal ZBP1 IF signal over the untransfected control (Fig. 2C,D). Further, depletion of MyoVa by shRNA resulted in a $61.5 \pm 20.4 \%$ increase in axonal ZBP1 levels compared to control shRNA-treated neurons (Fig. 2E,F). Quantification of ZBP1 signal in the cell bodies of transfected neurons indicated a $26.6 \pm 5.4 \%$ decrease in ZBP1 levels in the cell body, indicating that the accumulation of ZBP1 in neurites may be due to transport (Fig. 2E,F). Collectively, these data suggest that MyoVa may inhibit transport and accumulation of ZBP1 into axons. To examine whether the effect of MyoVa perturbation or knockdown on ZBP1 localization might also be apparent by disruption of F-actin, neurons were treated with cytochalasin D. ZBP1 levels in axons of cytochalasin D-treated cells were $54.1 \pm$ $11.91 \%$ higher as compared to the untreated cells (Fig. 2G,H), suggesting that F-actin and associated myosin $\mathrm{V}$ motors may limit ZBP1 localization and levels within the axon.

To investigate the role of MyoVa in ZBP1 transport, live cell imaging was performed on neurons transfected with mCherryZBP1. ZBP1 particles were dynamic and exhibited bidirectional transport with a bias in the retrograde orientation (Fig. $3 A, B)$. As reported previously, they displayed a maximum average speed of $0.1-2.0 \mu \mathrm{m} / \mathrm{s}$, consistent with the speed of fast microtubule (MT) and actin-based motors (Zhang et al., 2001). Inhibition of MyoVa by transduction with a GFP-GTD construct resulted in a 1.6-fold increase in the number of motile ZBP1 particles in the neurites (Fig. 3C). However, there was no alteration in the maximum average speed of the particles (data not shown). Analysis of orientation of motile particles in untransfected cells indicated a bias toward retrograde orientation (Fig. $3 D$ ). While mCherryZBP1 particles displayed a bias toward retrograde orientation at basal state, this was removed in GTDexpressing neurons where there was a concomitant increase in the anterograde oriented tracks (Fig. 3D). Inhibition of MyoVa also led to a moderate but significant $(14.9 \pm 4.4 \%)$ increase in the average run length of all motile ZBP1 particles, which included directed, bidirectional, and oscillatory particles (Fig. 3E). To further examine the effect on run lengths, the run lengths of all directed ZBP1 particles, both anterograde and retrograde, were compared. There was a marked increase in run lengths $(3.19 \pm$ 1.1-fold) in the anterograde orientation in the GFP-GTDtransduced cells (Fig. 3F). This suggests that MyoVa may be in-

$\leftarrow$

(Figure legend continued.) average fold-basal PAGFP-ZBP1 intensities in multiple neurons transfected with MyoVa shRNA or control shRNA as a function of time. $\boldsymbol{D}$, A model to depict the proposed role of myosin Va in ZBP1 transport. Myosin Va may tether ZBP1 on subcortical F-actin and compete with kinesin-mediated anterograde transport along microtubules. Myosin Va may regulate ZBP1 traffic by restraining anterograde transport in the cell body (1), along the axon (2), or within the growth cone (3). volved in limiting anterograde ZBP1 particle runs. To gather further evidence for the effect of myosin $\mathrm{Va}$ inhibition on orientation, we performed additional live cell experiments on PAGFPZBP1 expressed in neurons. PAGFP-ZBP1-expressing cells were selectively photoactivated in the cell body, and the accumulation of GFP signal in the neurites as a measure of anterograde transport was quantified in cells expressing MyoVa shRNA or control shRNA (Figure $4 A, B$ ). Cells transfected with MyoVa shRNA showed a 1.9-fold higher GFP signal accumulation in neurites by 5 min over control shRNA-transfected cells (Figure 4C), further confirming that inhibition of MyoVa leads to increased anterograde movement of ZBP1 and that MyoVa plays a restraining role on anterograde movement of ZBP1 from the cell body.

\section{Discussion}

The ability of a neuron to regulate steady-state expression and transport dynamics of mRNA granules in axons and dendrites is believed to be modulated by mRNA binding proteins acting as adapters for molecular motors. While previous work has shown that ZBP1-mediated localization of $\beta$-actin mRNA into axons depends on microtubules (Zhang et al., 1999, 2001), it remains unclear what molecular motors are involved in the directed transport of ZBP1. Kinesin motors have been shown to play an important role in the transport of mRNAs and mRNA binding proteins in neurons (Kanai et al., 2004; Dictenberg et al., 2008). In these studies, perturbation of kinesin leads to a reduction in RNA granule localization in neuronal processes and impaired dynamics. Here, we report that perturbation of myosin Va leads to an opposite phenotype, characterized by increased levels and transport dynamics of ZBP1 in axons. These findings suggest that transport dynamics for ZBP1 and perhaps other mRNA granule components are regulated by both microtubule- and actin-based motors. Our data indicate a novel role for MyoVa to regulate ZBP1 transport in axons. Previous work has shown that $\beta$-actin mRNA localization in the absence of MyoVa is altered (Salerno et al., 2008). However, the requirement of MyoVa to regulate the transport dynamics and axonal localization of an mRNA binding protein necessary for mRNA localization has not been previously shown.

Here we show that inhibition of MyoVa resulted in an overall increase in anterograde transport and accumulation of ZBP1 protein in axons, suggesting that MyoVa plays a restraining role on $\mathrm{ZBP} 1$ protein motility in the axon. The increased accumulation of ZBP1 in axons following perturbation or knockdown of MyoVa could be attributed to increased availability of ZBP1 for microtubule-dependent anterograde transport, perhaps resulting from release of ZBP1 from subcortical actin docking sites in the cell body or neuronal processes, resulting in a change in the frequency and dynamics of ZBP1 particles moving along microtubules at steady state. These results bear some similarities to other examples of MyoVa inhibition leading to an accumulation of cargoes, like the neurofilaments (Alami et al., 2009), where MyoVa regulates transport by decreasing the frequency of pauses. MyoVa is believed to be a short-range motor in neurons owing to the fact that F-actin filaments are small and of mixed polarity in neuronal processes. In the case of ZBP1, short-range tracks by MyoVa might serve to regulate the availability of ZBP1 for transport to the axon and ultimately the growth cone.

Although we have previously observed the bidirectional transport of ZBP1 in axons and growth cones (Zhang et al., 2001; Welshhans et al., 2011), here we analyze particle direction and reveal a retrograde bias of ZBP1 transport in axons. In Drosophila embryos, apically localized RNAs, which should move retro- 
gradely on MTs, do move in both directions; the resultant retrograde motility is only because of more frequent retrograde runs due to increased numbers of dynein motors (Bullock et al., 2006). These observations exemplify the tug-of-war hypothesis for bidirectional transport, where multiple motors similarly or oppositely oriented are present on a cargo, and the resultant direction of motility is a sum of forces. Multiple motors may be engaged in a tug of war on RNA or other cargoes, and the resultant orientation may be a function of the number of the anterograde or retrograde motors (Holzbaur and Goldman, 2010). While MTbased motors are likely the primary transporters along neuronal processes, the role of MyoVa might be to tether or limit interactions with microtubule-based motors. We speculate that in neurons MyoVa may facilitate a retrograde orientation of ZBP1 particles by tipping the balance to a retrograde motor like dynein (Fig. 4D). Inhibition of MyoVa by expressing a dominant negative thus leads to more frequent and longer anterograde runs. This may or may not involve direct interactions with dynein, since it is also suggested that MyoVa and dynein share the same light chains (Espindola et al., 2000; Hódi et al., 2006). Functionally, this may be significant for axon guidance, since RNA binding proteins like ZBP1 are negative regulators of their target RNA translation, and an additional control to keep the translation of key molecules dormant would be to restrict their transport unless required. Retrograde transport may also facilitate retrograde signaling or recycling of ZBP1.

ZBP1 may associate with MyoVa directly and independently of RNA. Thus, RNA binding proteins like ZBP1 may be considered as adapters for RNA to associate with motors. The mechanism that governs MyoVa association remains unknown. Phosphorylation at Y396 stimulates release of ZBP1 from $\beta$-actin mRNA (Hüttelmaier et al., 2005), but does not alter ZBP1 binding to MyoVa (data not shown). There may be other posttranslational modifications that determine the binding of ZBP1 to MyoVa. It is also likely that post-translational modifications on MyoVa itself may regulate ZBP1 binding. It is known that phosphorylation, calcium, and cargo binding can alter the motility of MyoVa (Krementsov et al., 2004; Sellers and Knight, 2007). However, it has been found recently, using in vitro and in vivo single-molecule RNA imaging, that localization elements in the RNA were sufficient to decide the fate of localized RNA by increasing the number of dynein-dynactin recruited on the RNA (Amrute-Nayak and Bullock, 2012).

In summary, our results suggest a model (Fig. 4D) whereby MyoVa plays a restraining role for ZBP1 transport to and from axons and growth cones by regulating recruitment into the neurite and run lengths along microtubules. Like other neuronal cargoes, motility along the neurite may involve predominantly MT-based motors. We still do not know what types of MT-based motors may be involved. Both KIF1 and KIF5 motors are involved in the transport Fragile X mental retardation protein (Davidovic et al., 2007; Dictenberg et al., 2008), and kinesins, dynein and myosin motors are all bound to localized RNAs like oskar in Drosophila (Bullock, 2011). Further work is needed to identify other motors and elucidate their cooperative involvement in ZBP1 transport and its regulation by physiological signals, which will lead to a clearer understanding of motor dynamics during basal and activated states.

\section{Notes}

Supplemental material for this article is available at http://basselllab.com/ Nalavadi/. The URL contains two movie files showing the motility of mCherry
ZBP1 particles in untransduced and GTD transduced neurons and a text file with a legend This material has not been peer reviewed.

\section{References}

Alami NH, Jung P, Brown A (2009) Myosin Va increases the efficiency of neurofilament transport by decreasing the duration of long-term pauses. J Neurosci 29:6625-6634. CrossRef Medline

Ali MY, Kennedy GG, Safer D, Trybus KM, Sweeney HL, Warshaw DM (2011) Myosin Va and myosin VI coordinate their steps while engaged in an in vitro tug of war during cargo transport. Proc Natl Acad Sci U S A 108:E535-541. CrossRef Medline

Amrute-Nayak M, Bullock SL (2012) Single-molecule assays reveal that RNA localization signals regulate dynein-dynactin copy number on individual transcript cargoes. Nat Cell Biol 14:416-423. CrossRef Medline

Bullock SL (2011) Messengers, motors and mysteries: sorting of eukaryotic mRNAs by cytoskeletal transport. Biochem Soc Trans 39:1161-1165. CrossRef Medline

Bullock SL, Nicol A, Gross SP, Zicha D (2006) Guidance of bidirectional motor complexes by mRNA cargoes through control of dynein number and activity. Curr Biol 16:1447-1452. CrossRef Medline

Davidovic L, Jaglin XH, Lepagnol-Bestel AM, Tremblay S, Simonneau M, Bardoni B, Khandjian EW (2007) The fragile X mental retardation protein is a molecular adaptor between the neurospecific KIF3C kinesin and dendritic RNA granules. Hum Mol Genet 16:3047-3058. CrossRef Medline

Dictenberg JB, Swanger SA, Antar LN, Singer RH, Bassell GJ (2008) A direct role for FMRP in activity-dependent dendritic mRNA transport links filopodial-spine morphogenesis to fragile X syndrome. Dev Cell 14:926939. CrossRef Medline

Espindola FS, Suter DM, Partata LB, Cao T, Wolenski JS, Cheney RE, King SM, Mooseker MS (2000) The light chain composition of chicken brain myosin-Va: calmodulin, myosin-II essential light chains, and 8-kDa dynein light chain/PIN. Cell Motil Cytoskeleton 47:269-281. CrossRef Medline

Hendricks AG, Perlson E, Ross JL, Schroeder HW 3rd, Tokito M, Holzbaur EL (2010) Motor coordination via a tug-of-war mechanism drives bidirectional vesicle transport. Curr Biol 20:697-702. CrossRef Medline

Hódi Z, Németh AL, Radnai L, Hetényi C, Schlett K, Bodor A, Perczel A, Nyitray L (2006) Alternatively spliced exon B of myosin Va is essential for binding the tail-associated light chain shared by dynein. Biochemistry 45:12582-12595. CrossRef Medline

Holzbaur EL, Goldman YE (2010) Coordination of molecular motors: from in vitro assays to intracellular dynamics. Curr Opin Cell Biol 22:4-13. CrossRef Medline

Hüttelmaier S, Zenklusen D, Lederer M, Dictenberg J, Lorenz M, Meng X, Bassell GJ, Condeelis J, Singer RH (2005) Spatial regulation of $\beta$-actin translation by Src-dependent phosphorylation of ZBP1. Nature 438:512515. CrossRef Medline

Kanai Y, Dohmae N, Hirokawa N (2004) Kinesin transports RNA: isolation and characterization of an RNA-transporting granule. Neuron 43: 513-525. CrossRef Medline

Kiebler MA, Bassell GJ (2006) Neuronal RNA granules: movers and makers. Neuron 51:685-690. CrossRef Medline

Kögel T, Bittins CM, Rudolf R, Gerdes HH (2010) Versatile roles for myosin $\mathrm{Va}$ in dense core vesicle biogenesis and function. Biochem Soc Trans 38:199-204. CrossRef Medline

Krauss J, López de Quinto S, Nüsslein-Volhard C, Ephrussi A (2009) Myosin-V regulates oskar mRNA localization in the Drosophila oocyte. Curr Biol 19:1058-1063. CrossRef Medline

Krementsov DN, Krementsova EB, Trybus KM (2004) Myosin V: regulation by calcium, calmodulin, and the tail domain. J Cell Biol 164:877-886. CrossRef Medline

Lewis TL Jr, Mao T, Svoboda K, Arnold DB (2009) Myosin-dependent targeting of transmembrane proteins to neuronal dendrites. Nat Neurosci 12:568-576. CrossRef Medline

Li XD, Jung HS, Mabuchi K, Craig R, Ikebe M (2006) The globular tail domain of myosin Va functions as an inhibitor of the myosin Va motor. J Biol Chem 281:21789-21798. CrossRef Medline

Martin KC, Ephrussi A (2009) mRNA localization: gene expression in the spatial dimension. Cell 136:719-730. CrossRef Medline

Muddashetty RS, Kelić S, Gross C, Xu M, Bassell GJ (2007) Dysregulated metabotropic glutamate receptor-dependent translation of AMPA recep- 
tor and postsynaptic density-95 mRNAs at synapses in a mouse model of fragile X syndrome. J Neurosci 27:5338-5348. CrossRef Medline

Narayanan U, Nalavadi V, Nakamoto M, Pallas DC, Ceman S, Bassell GJ, Warren ST (2007) FMRP phosphorylation reveals an immediate-early signaling pathway triggered by group I mGluR and mediated by PP2A. J Neurosci 27:14349-14357. CrossRef Medline

Pastural E, Barrat FJ, Dufourcq-Lagelouse R, Certain S, Sanal O, Jabado N, Seger R, Griscelli C, Fischer A, de Saint Basile G (1997) Griscelli disease maps to chromosome $15 \mathrm{q} 21$ and is associated with mutations in the myosin-Va gene. Nat Genet 16:289-292. CrossRef Medline

Pathak D, Sepp KJ, Hollenbeck PJ (2010) Evidence that myosin activity opposes microtubule-based axonal transport of mitochondria. J Neurosci 30:8984-8992. CrossRef Medline

Rao MV, Engle LJ, Mohan PS, Yuan A, Qiu D, Cataldo A, Hassinger L, Jacobsen S, Lee VM, Andreadis A, Julien JP, Bridgman PC, Nixon RA (2002) Myosin Va binding to neurofilaments is essential for correct myosin Va distribution and transport and neurofilament density. J Cell Biol 159:279-290. CrossRef Medline

Ross AF, Oleynikov Y, Kislauskis EH, Taneja KL, Singer RH (1997) Characterization of a $\beta$-actin mRNA zipcode-binding protein. Mol Cell Biol 17:2158-2165. Medline

Ross JL, Shuman H, Holzbaur EL, Goldman YE (2008) Kinesin and dyneindynactin at intersecting microtubules: motor density affects dynein function. Biophys J 94:3115-3125. CrossRef Medline

Salerno VP, Calliari A, Provance DW Jr, Sotelo-Silveira JR, Sotelo JR, Mercer JA (2008) Myosin-Va mediates RNA distribution in primary fibroblasts from multiple organs. Cell Motil Cytoskeleton 65:422-433. CrossRef Medline

Sasaki Y, Welshhans K, Wen Z, Yao J, Xu M, Goshima Y, Zheng JQ, Bassell GJ (2010) Phosphorylation of zipcode binding protein 1 is required for brain-derived neurotrophic factor signaling of local beta-actin synthesis and growth cone turning. J Neurosci 30:9349-9358. CrossRef Medline
Sellers JR, Knight PJ (2007) Folding and regulation in myosins II and V. J Muscle Res Cell Motil 28:363-370. CrossRef Medline

Takagishi Y, Murata Y (2006) Myosin Va mutation in rats is an animal model for the human hereditary neurological disease, Griscelli syndrome type 1. Ann NY Acad Sci 1086:66-80. CrossRef Medline

Tamada A, Kawase S, Murakami F, Kamiguchi H (2010) Autonomous right-screw rotation of growth cone filopodia drives neurite turning. J Cell Biol 188:429-441. CrossRef Medline

Wagner W, Brenowitz SD, and Hammer JA 3rd (2011) Myosin-Va transports the endoplasmic reticulum into the dendritic spines of Purkinje neurons. Nat Cell Biol 13:40-48. CrossRef Medline

Welshhans K, Bassell GJ (2011) Netrin-1-induced local $\beta$-actin synthesis and growth cone guidance requires zipcode binding protein 1. J Neurosci 31:9800-9813. CrossRef Medline

Wu X, Bowers B, Wei Q, Kocher B, Hammer JA 3rd (1997) Myosin V associates with melanosomes in mouse melanocytes: evidence that myosin $\mathrm{V}$ is an organelle motor. J Cell Sci 110:847-859. Medline

Yao J, Sasaki Y, Wen Z, Bassell GJ, Zheng JQ (2006) An essential role for beta-actin mRNA localization and translation in $\mathrm{Ca}^{2+}$-dependent growth cone guidance. Nat Neurosci 9:1265-1273. CrossRef Medline

Yoshimura A, Fujii R, Watanabe Y, Okabe S, Fukui K, Takumi T (2006) Myosin-Va facilitates the accumulation of mRNA/protein complex in dendritic spines. Curr Biol 16:2345-2351. CrossRef Medline

Zhang HL, Singer RH, Bassell GJ (1999) Neurotrophin regulation of betaactin mRNA and protein localization within growth cones. J Cell Biol 147:59-70. CrossRef Medline

Zhang HL, Eom T, Oleynikov Y, Shenoy SM, Liebelt DA, Dictenberg JB, Singer RH, Bassell GJ (2001) Neurotrophin-induced transport of a betaactin mRNP complex increases beta-actin levels and stimulates growth cone motility. Neuron 31:261-275. CrossRef Medline 